ESAIM: PROCEEDINGS, April 2012, Vol. 36, p. 197-208

Danièle Fournier-Prunaret, Laura Gardini, \& Ludwig Reich, Editors

\title{
ON THE HELIX EQUATION
}

\section{Mohamed Hmissi ${ }^{1}$, Imene Ben Salah ${ }^{2}$ and Hajer TaOuil ${ }^{3}$}

\begin{abstract}
This paper is devoted to the helices processes, i.e. the solutions $H: \mathbb{R} \times \Omega \rightarrow \mathbb{R}^{d},(t, \omega) \mapsto$ $H(t, \omega)$ of the helix equation

$$
H(0, \omega)=0 ; \quad H(s+t, \omega)=H(s, \Phi(t, \omega))+H(t, \omega)
$$

where $\Phi: \mathbb{R} \times \Omega \rightarrow \Omega,(t, \omega) \mapsto \Phi(t, \omega)$ is a dynamical system on a measurable space $(\Omega, \mathcal{F})$.

More precisely, we investigate dominated solutions and non differentiable solutions of the helix equation. For the last case, the Wiener helix plays a fundamental role. Moreover, some relations with the cocycle equation defined by $\Phi$, are investigated.
\end{abstract}

MSC (2000): Primary: 39B52; Secondary, 37B50, 37H10, 60H25

Key words: translation equation, helix equation, Wiener helix, cocycle equation.

Résumé. Ce papier est consacré aux hélices, c'est-à-dire les solutions $H: \mathbb{R} \times \Omega \rightarrow \mathbb{R}^{d},(t, \omega) \mapsto H(t, \omega)$ de l'équation fonctionnelle

$$
H(0, \omega)=0 ; \quad H(s+t, \omega)=H(s, \Phi(t, \omega))+H(t, \omega)
$$

où $\Phi: \mathbb{R} \times \Omega \rightarrow \Omega,(t, \omega) \mapsto \Phi(t, \omega)$ est un système dynamique défini sur un espace mesurable $(\Omega, \mathcal{F})$. Plus présisément, nous déterminons d'abord les hélices dominées puis nous caractérisons les hélices non différentiables. Dans ce dernier cas, l'hélice de Wiener joue un rôle important. Nous précisons aussi quelques relations des hélices avec les cocycles définis par $\Phi$.

Mots clefs: Equation de translation, Equation d'hélice, Héelice de Wiener, Cocycle.

\section{INTRODUCTION}

Let $(\Omega, \mathcal{F})$ be a measurable space and let $\Phi: \mathbb{R} \times \Omega \rightarrow \Omega$ be a dynamical system on $(\Omega, \mathcal{F})$, that is $\Phi$ satisfies the translation equation

$$
\Phi(0, \omega)=\omega, \quad \Phi(s+t, \omega)=\Phi(s, \Phi(t, \omega))
$$

\footnotetext{
1 Département de Mathématiques, Faculté des Sciences de Tunis, Université de Tunis Elmanar, TN-2092 Elmanar, Tunis, Tunisia; e-mail: Med.Hmissi@fst.rnu.tn.

2 Département de Mathématiques, Faculté des Sciences de Tunis, Université de Tunis Elmanar, TN-2092 Elmanar, Tunis, Tunisia; e-mail: bensalah_imene@live.fr.

3 Département de Mathématiques, Faculté des Sciences de Tunis, Université de Tunis Elmanar, TN-2092 Elmanar, Tunis, Tunisia; e-mail: hajertaouil@yahoo.fr. 
A $\Phi$-helix (or helix over $\Phi$ ) on $\mathbb{R}^{d}$, is a mapping $H: \mathbb{R} \times \Omega \rightarrow \mathbb{R}^{d}$ which satisfies the helix equation

$$
H(0, \omega)=0, \quad H(s+t, \omega)=H(s, \Phi(t, \omega))+H(t, \omega) .
$$

An helix is always denoted by $(\Omega, \mathcal{F}, \Phi, H)$.

This notion was first introduced by Kolmogorov [7] in the framework of stochastic integrals and stochastic differential equations (cf. also [2] and the related references, for more details).

The aim of this paper, is to investigate helices as functional equations.

In the first part, we suppose that $H$ is dominated, i.e. there exists two constants $a \geq 0, b \geq 0$ such that (|.| denotes a norm on $\mathbb{R}^{d}$ )

$$
\left|H_{t}(\omega)\right| \leq a t+b ; \quad(\omega \in \Omega, t \geq 0)
$$

Using a result in [6], we prove that $H$ is a sum of a closed $\Phi$-helix and a differentiable one, i.e. there exists $g, h: \Omega \rightarrow \mathbb{R}^{d}$ measurable such that $s \mapsto g(\Phi(s, \omega))$ is locally integrable and

$$
H(t, \omega)=h(\omega)-h\left(\Phi_{t} \omega\right)+\int_{0}^{t} g(\Phi(s, \omega)) d s .
$$

However, the Wiener helix denoted by $\left(\Omega_{0}, \mathcal{F}_{0}, \Theta, W\right)$ is neither closed nor differentiable (cf. 3.1 below for more details). This is why, we investigate the general case in the second part of this paper.

We prove first that, if $H$ separates points of $\Omega$ and generates $\mathcal{F}$, then the associated dynamical system is uniquely determined.

Then we prove that, each helix $(\Omega, \mathcal{F}, \Phi, H)$ is conjugate to the Wiener helix $\left(\Omega_{0}, \mathcal{F}_{0}, \Theta, W\right)$, i.e there exists a mapping $\kappa: \Omega \rightarrow \Omega_{0}$ such that

$$
\kappa(\Phi(t, \omega))=\Theta(t, \kappa(\omega)) ; \quad H(t, \omega)=W(t, \kappa(\omega)) .
$$

A $\Phi$-helix $H$ is said to be connecting if, for all $x, y \in \mathbb{R}^{d}$ there exists $\omega \in \Omega, s, t \in \mathbb{R}$ such that $x=H(s, \omega)$ and $y=H(t, \omega)$. After giving some examples of connecting and non connecting helices, we prove the following result: The image of a connecting $\Phi$-helix by a Borel function $f: \mathbb{R}^{d} \rightarrow \mathbb{R}^{n}$, is a $\Phi$-helix if and only if $f$ is linear. The proof is in fact a consequence of the well known results about the Cauchy equation. We close the paper by some classical relations between helices and cocycles. Moreover, we introduce the notion of bi-helix.

This paper is a continuation of our paper [5] which is devoted to homogeneous process instead of helices processes, and some general notions introduced here, are picked from [5].

\section{Helix PROCESSES}

In this paper, we denote by $\mathbb{T}:=\mathbb{Z}$ or $\mathbb{R}$. If $\mathbb{T}=\mathbb{Z}$, we take $\mathcal{B}:=\mathcal{P}(\mathbb{Z})$ the $\sigma$-algebra of all subsets of $\mathbb{Z}$. If $\mathbb{T}=\mathbb{R}$, we take $\mathcal{B}:=\mathcal{B}(\mathbb{R})$ the Borel $\sigma$-algebra of $\mathbb{R}$. The space $\mathbb{R}^{d}, d \geq 1$ is also endowed with its Borel $\sigma$-algebra $\mathcal{B}_{d}$.

Let $(\Omega, \mathcal{F})$ be a measurable space. Each mapping $Y: \Omega \rightarrow \mathbb{R}^{d}$ which is $\left(\mathcal{F}, \mathcal{B}_{d}\right)$-measurable, is called random vector.

A stochastic process over $(\Omega, \mathcal{F})$ with state space $\mathbb{R}^{d}$ is a mapping $X: \mathbb{T} \times \Omega \rightarrow \mathbb{R}^{d}$ such that, for each $t \in \mathbb{T}$, the map $\omega \mapsto X(t, \omega)$ is a random vector.

The product space $\mathbb{T} \times \Omega$ is endowed with the product $\sigma$-algebra $\mathcal{B} \otimes \mathcal{F}$. If $X$ is $\left(\mathcal{B} \otimes \mathcal{F}, \mathcal{B}_{d}\right)$-measurable then the stochastic process is said to be measurable (Notice that if $\mathbb{T}=\mathbb{Z}$, each stochastic process is measurable). 


\subsection{Dynamical system}

A Dynamical System (notation DS) on $(\Omega, \mathcal{F})$, is a map $\Phi: \mathbb{T} \times \Omega \rightarrow \Omega,(t, \omega) \mapsto \Phi(t, \omega)$ such that

(1) $\omega \mapsto \Phi(t, \omega)$ is measurable for each $t \in \mathbb{T}$.

(2) $\Phi$ satisfies the translation equation, i.e. for all $\omega \in \Omega ; t, s \in \mathbb{T}$

$$
\Phi(0, \omega)=\omega ; \quad \Phi(s+t, \omega)=\Phi(s, \Phi(t, \omega)) .
$$

If $(t, \omega) \mapsto \Phi(t, \omega)$ is $(\mathcal{B} \otimes \mathcal{F}, \mathcal{F})$-measurable, the DS si said to be measurable.

If we put $\Phi_{t}:=\Phi(t,$.$) then the DS \Phi$ is identified with a family $\Phi:=\left(\Phi_{t}\right)_{t \in \mathbb{T}}$ of measurable transformations $\Phi_{t}: \Omega \rightarrow \Omega$ such that $\left(I_{\Omega}\right.$ denotes the identity operator on $\Omega$ )

$$
\Phi_{0}=I_{\Omega} ; \quad \Phi_{s+t}=\Phi_{s} \circ \Phi_{t} ; \quad(s, t \in \mathbb{T}) .
$$

The DS will be sometimes denoted by $(\Omega, \mathcal{F}, \Phi)$.

Let $(\Omega, \mathcal{F}, \Phi)$ be a DS. A measurable subset $\Gamma$ of $\Omega$ is said to be $\Phi$-invariant if $\Phi_{t}(\Gamma) \subset \Gamma$ for each $t \in \mathbb{T}$. In this case, the restriction of $\Phi$ to $\mathbb{T} \times \Gamma$ defines a so called (measurable) sub-dynamical system of $(\Omega, \mathcal{F}, \Phi)$.

Let $(\Omega, \mathcal{F}, \Phi)$ and $(\Upsilon, \mathcal{G}, \Psi)$ be two DS. A measurable map $f: \Omega \rightarrow \Upsilon$ is called SD-morphism if

$$
f\left(\Phi_{t}(\omega)\right)=\Psi_{t}(f(\omega)) ; \quad(t \in \mathbb{T}, \omega \in \Omega) .
$$

If $f$ is one-to-one and if $f$ and $f^{-1}$ are both SD-morphisms, we say that $f$ is an SD-isomorphism. In this case, the DS $(\Omega, \mathcal{F}, \Phi)$ and $(\Upsilon, \mathcal{G}, \Psi)$ will be conjugate (cf. [4] for example).

The following Lemma will be used later. The proof is classical (cf. [4] for example) and it is omitted.

Lemma 1 Let $(\Omega, \mathcal{F}, \Phi)$ and $(\Upsilon, \mathcal{G}, \Psi)$ be two $D S$ and let $f: \Omega \rightarrow \Upsilon$ be an injective $S D$ - morphism. Then the restriction of $\Psi$ to $f(\Omega)$ defines a sub-dynamical system of $(\Upsilon, \mathcal{G}, \Psi)$.

\subsection{Helix}

Let $(\Omega, \mathcal{F}, \Phi)$ be a dynamical system. A $\Phi$-helix on $\mathbb{R}^{d}$, is a mapping $H: \mathbb{T} \times \Omega \rightarrow \mathbb{R}^{d}$ such that

(1) $\omega \mapsto H(t, \omega)$ is measurable for each $t \in \mathbb{T}$.

(2) $H$ satisfies the helix equation, i.e. for all $\omega \in \Omega ; t, s \in \mathbb{T}$

$$
H(0, \omega)=0 ; \quad H(s+t, \omega)=H(s, \Phi(t, \omega))+H(t, \omega) .
$$

If $(t, \omega) \mapsto H(t, \omega)$ is $\left(\mathcal{B} \otimes \mathcal{F}, \mathcal{B}_{d}\right)$-measurable, the $\Phi$-helix $H$ is said to be measurable.

If we put $H_{t}:=H(t,$.$) , then the \Phi$-helix $H$ is identified to a stochastic process $H:=\left(H_{t}\right)_{t \in \mathbb{T}}$ such that

$$
H_{0}=0, \quad H_{s+t}=H_{s} \circ \Phi_{t}+H_{t} \quad(s, t \in \mathbb{T}) .
$$

\section{Remarks 1}

(1) If $U, V$ are two $\Phi$-helices and if $a, b \in \mathbb{R}$ then, $a U+b V$ is a $\Phi$-helix.

(2) If we write $H_{t}=\left(H_{t}^{1}, \ldots, H_{t}^{d}\right)$, then $H=\left(H_{t}\right)_{t \in \mathbb{T}}$ is a $\Phi$-helix on $\mathbb{R}^{d}$ if and only if, for each $1 \leq k \leq d$, $H^{k}=\left(H_{t}^{k}\right)_{t \in \mathbb{T}}$ is a real $\Phi$-helix.

(3) Let $(\Omega, \mathcal{F}, \Phi)$ and $(\Upsilon, \mathcal{G}, \Psi)$ be two DS and let $f: \Omega \rightarrow \Upsilon$ be an SD- morphism. If $H=\left(H_{t}\right)_{t \in \mathbb{T}}$ is a $\Psi$-helix, then $H \circ f:=\left(H_{t} \circ f\right)_{t \in \mathbb{T}}$ is a $\Phi$-helix. $H \circ f$ is said to be conjugate to $H$. 
(4) In particular, if $(\Omega, \mathcal{F}, \Phi)$ is a DS and if $\Gamma$ is a $\Phi$-invariant subset of $\Omega$, then the restriction to $\mathbb{T} \times \Gamma$ of each helix, is an helix for the associated sub-dynamical system.

(5) Let $H$ be a $\Phi$-helix. From the helix equation (3), we deduce that

$$
H_{t}=-H_{-t} \circ \Phi_{t} ; \quad(t \in \mathbb{T})
$$

This relation will be used as the following: If $H_{t}$ is given for $t \geq 0$, it will completely determined for all $t \in \mathbb{T}$.

\subsection{The discrete case}

If $T=\mathbb{Z}$ then $\Phi:=\left(\Phi_{t}\right)_{t \in \mathbb{Z}}$ is generated by $\phi:=\Phi_{1}$. In this case, an helix $H$ is considered as a $\phi$-helix. Moreover, the helix equation (3) is equivalent to the iteration equation

$$
H_{0}=0 ; \quad H_{n+1}=H_{1} \circ \phi^{n}+H_{n} ; \quad(n \in \mathbb{Z}) .
$$

Let $h:=H_{1}$. By an easy induction in (5), we obtain

$$
H_{n}= \begin{cases}\sum_{k=0}^{n-1} h \circ \phi^{k} & ;(n \geq 1) \\ 0 & ;(n=0) \\ -\sum_{k=1}^{n} h \circ \phi^{-k} & ;(n \leq-1) .\end{cases}
$$

Conversely, for any random vector $h: \Omega \rightarrow \mathbb{R}^{d}$, formula (6) defines a measurable $\phi$-helix.

\section{Dominated helices}

\subsection{Closed helices}

Let $\Phi$ be a DS defined on a measurable space $(\Omega, \mathcal{F})$ and let $h: \Omega \rightarrow \mathbb{R}^{d}$ be random vector, then

$$
H_{t}(\omega):=h(\omega)-h\left(\Phi_{t} \omega\right) ; \quad(t \in \mathbb{R}, \omega \in \Omega)
$$

defines a $\Phi$-helix. Such a $\Phi$-helix is said to be closed.

Conversely, we have the following partial result:

Let $H:=\left(H_{t}\right)_{t \in \mathbb{R}}$ be a real $\Phi$-helix such that

$$
(t \geq 0) \quad \Rightarrow \quad\left(H_{t}(\omega) \geq 0 \text { for each } \omega \in \Omega\right)
$$

Note first that by (4), the condition (8) is equivalent to

$$
(t \leq 0) \quad \Rightarrow \quad\left(H_{t}(\omega) \leq 0 \text { for each } \omega \in \Omega\right)
$$

In this case, $H(., \omega):[0, \infty[\rightarrow \mathbb{R}$ is increasing. Letting $s \rightarrow+\infty$ in $(3)$, we obtain

$$
\sup H_{s}(\omega)=\sup H_{s}\left(\Phi_{t} \omega\right)+H_{t}(\omega) \text {. }
$$

Hence $H$ will be closed if

$$
h(\omega):=\sup H_{s}(\omega)<\infty ; \quad(\omega \in \Omega)
$$


This result can be easily generalized to a $\Phi$-helix $H$ on $\mathbb{R}^{d}$ such that each component of $H$ satisfies (8) and (10).

Remark 2 Suppose that (7) is verified for $t \geq 0$ and for some random vector $h$. Then for $t \leq 0$, (4) and (7) imply that

$$
H_{t}(\omega)=-H_{-t}\left(\Phi_{t} \omega\right)=-\left[h\left(\Phi_{t} \omega\right)-h\left(\Phi_{-t} \Phi_{t} \omega\right)\right]=h(\omega)-h\left(\Phi_{t} \omega\right)
$$

Hence (7) is satisfied for the same $h$ and for all $t \in \mathbb{R}$.

\subsection{Differentiable helices}

Let $\Phi$ be a DS defined on a measurable space $(\Omega, \mathcal{F})$ and $g: \Omega \rightarrow \mathbb{R}^{d}$ a random vector such that $t \mapsto g\left(\Phi_{t}(\omega)\right)$ is locally integrable for each $\omega \in \Omega$, then

$$
H_{t}(\omega):=\int_{0}^{t} g\left(\Phi_{r}(\omega)\right) d r ; \quad(t \in \mathbb{R}, \omega \in \Omega)
$$

defines a so called differentiable $\Phi$-helix.

Remark 3 Suppose that (11) is true for $t \geq 0$ and for some random vector $g$. Let $t \leq 0$. Using (1), (4) and (11) we deduce

$$
\begin{array}{r}
H_{t}(\omega)=-H_{-t}\left(\Phi_{t} \omega\right)=-\left[\int_{0}^{t} g\left(\Phi_{r} \Phi_{t} \omega\right) d r\right] \\
=-\left[\int_{0}^{t} g\left(\Phi_{r+t} \omega\right) d r\right]=\int_{0}^{t} g\left(\Phi_{r}(\omega)\right) d r
\end{array}
$$

In the same way, (11) is also satisfied for the same $g$ and for all $t \in \mathbb{R}$.

Definition $1 \mathrm{~A} \Phi$-helix $H:=\left(H_{t}\right)_{t \in \mathbb{R}}$ is said to be dominated if there exists two constants $a \geq 0, b \geq 0$ such that $\left(|\cdot|\right.$ denotes a norm on $\left.\mathbb{R}^{d}\right)$

$$
\left|H_{t}(\omega)\right| \leq a t+b ; \quad(\omega \in \Omega, t \geq 0)
$$

Theorem 1 Let $H: \mathbb{R} \times \Omega \rightarrow \mathbb{R}$ be a dominated $\Phi$-helix. Then $H$ is the sum of a closed $\Phi$-helix and a differentiable one.

Proof. Put

$$
L_{t}(\omega):=\exp \left(H_{t}(\omega)\right) ; \quad(t \geq 0, \omega \in \Omega)
$$

Then the helix equation becomes

$$
L_{s+t}(\omega)=L_{t}(\omega) \cdot L_{s}\left(\Phi_{t} \omega\right) ; \quad(s, t \geq 0, \omega \in \Omega)
$$

Hence $L$ is a real cocycle in the sens of [6] (Definition 1.3). Moreover, (12) implies that $L$ is dominated in the sens of [6] (Definition 1.5). Consequently, there exists $q: \Omega \rightarrow] 0, \infty\left[; f: \Omega \rightarrow \mathbb{R}\right.$ such that $t \mapsto f\left(\Phi_{t} \omega\right)$ is locally integrable and

$$
L_{t}(\omega)=\frac{q\left(\Phi_{t} \omega\right)}{q(\omega)} \cdot \exp \left(\int_{0}^{t} f\left(\Phi_{s} \omega\right) d s\right) ; \quad(t \geq 0, \omega \in \Omega)
$$


This formula is proved by the first author in [6] (cf. Proposition 2.2).

Let $U_{t}(\omega):=\ln \left(q\left(\Phi_{t} \omega\right)\right)-\ln (q(\omega))$ and $V_{t}(\omega):=\int_{0}^{t} f\left(\Phi_{s} \omega\right) d s$ for $t \geq 0$ and $\omega \in \Omega$. By (13) and (15) we have

$$
H_{t}(\omega)=U_{t}(\omega)+V_{t}(\omega) ; \quad(\omega \in \Omega, t \geq 0) .
$$

However, using (4), Remark 2 and Remark 3, the preceding formula (16) holds for all $t \in \mathbb{R}$. Therefore, $U$ is a closed $\Phi$-helix, $V$ is a differentiable $\Phi$-helix and $H=U+V$.

Corollary 1 Each dominated $\Phi$-helix $H: \mathbb{R} \times \Omega \rightarrow \mathbb{R}^{d}$ is a sum of a closed $\Phi$-helix and a differentiable one.

Proof. We write $H_{t}=\left(H_{t}^{1}, \ldots, H_{t}^{d}\right)$. Since $H$ is dominated, then each of its components is also a dominated real $\Phi$-helix (cf. Remark 1.2). We conclude by the preceding result.

\section{NON-DiffEREnTIABLE HeliCES}

Let $(\Omega, \mathcal{F})$ be a measurable space. If $X:=\left(X_{t}\right)_{t \in \mathbb{R}}$ is a stochastic process on $\mathbb{R}^{d}$, we denote by $\sigma\left(X_{t}: t \in \mathbb{R}\right)$ the smallest $\sigma$-algebra on $\Omega$ for which each $X_{t}$ is measurable. If $\mathcal{F}=\sigma\left(X_{t}: t \in \mathbb{R}\right)$, we say that $X$ generates $\mathcal{F}$. The stochastic process $X$ separates the points of $\Omega$, if for $\omega_{1}, \omega_{2} \in \Omega$, the equality $X_{t}\left(\omega_{1}\right)=X_{t}\left(\omega_{2}\right)$ for each $t \in \mathbb{R}$ implies that $\omega_{1}=\omega_{2}$.

We give first a little more general definition of helix.

Definition 2 An helix over $(\Omega, \mathcal{F})$ is a stochastic process on $\mathbb{R}^{d}$ such that there exists a family $\Phi:=\left(\Phi_{t}\right)_{t \in \mathbb{R}}$ of measurable transformations $\Phi_{t}: \Omega \rightarrow \Omega$ satisfying (3), i.e.

$$
H_{0}=0, \quad H_{s+t}=H_{s} \circ \Phi_{t}+H_{t} \quad(s, t \in \mathbb{R}) .
$$

Under some natural assumptions, the family of transformations $\Phi:=\left(\Phi_{t}\right)_{t \in \mathbb{R}}$ which arises in (17), is in fact a dynamical system. This is the subject of the next result.

Proposition 1 Let $H:=\left(H_{t}\right)_{t \in \mathbb{R}}$ be an helix which separates points of $\Omega$ and generates $\mathcal{F}$. Then, there exists a unique DS $\Phi$ such that (17) holds.

Proof. Since $H$ is an helix process, then there exists $\Phi$ such that (17) holds. Moreover, $\Phi$ is uniquely determined because $H$ is separating. From (17), we have $H_{s}=H_{s} \Phi_{0}$ for each $s \in \mathbb{R}$, hence $\Phi_{0}=I_{\Omega}$ since $H$ separates the points of $\Omega$. Let $r, s, t \in \mathbb{R}$; by iteration of (17), we have

$$
\begin{aligned}
H_{r} o \Phi_{s} o \Phi_{t} & =\left(H_{r} o \Phi_{s}\right) o \Phi_{t} \\
& =\left(H_{r+s}-H_{s}\right) o \Phi_{t} \\
& =H_{r+s} \Phi_{t}-H_{s} o \Phi_{t} \\
& =H_{r+s+t}-H_{t}-H_{s+t}+H_{t} \\
& =H_{r} \Phi_{s+t} .
\end{aligned}
$$

This implies the group property (1) since $H$ separates the points of $\Omega$.

Let $t, t_{1}<\ldots<t_{n} \in \mathbb{R} ; A_{1}, \ldots, A_{n} \in \mathcal{B}_{d}$. From the helix relation (17), we have (using the notation $\left[H_{t} \in A\right]:=$ $\left.\left\{\omega \in \Omega: H_{t}(\omega) \in A\right\}\right)$

$$
\Phi_{-t}\left[H_{t_{1}} \in A_{1}, \ldots, H_{t_{n}} \in A_{n}\right]=\left[\left(H_{t_{1}+t}-H_{t}\right) \in A_{1}, \ldots,\left(H_{t_{n}+t}-H_{t}\right) \in A_{n}\right]
$$

which is in $\mathcal{F}$ since $\mathcal{F}=\sigma\left(H_{s}: s \in \mathbb{R}\right)$. Therefore each $\Phi_{t}$ is measurable. 


\subsection{Wiener helix}

Let $\Omega_{0}:=\left\{u: \mathbb{R} \rightarrow \mathbb{R}^{d}\right.$ such that $\left.u(0)=0\right\}$.

For each $t \in \mathbb{R}$, let $W_{t}: \Omega_{0} \rightarrow \mathbb{R}^{d}$ defined by

$$
W_{t}(u)=u(t) ; \quad\left(t \in \mathbb{R}, u \in \Omega_{0}\right)
$$

and let $\mathcal{F}_{0}=\sigma\left(W_{t}: t \in \mathbb{R}\right)$. Obviously $W:=\left(W_{t}\right)_{t \in \mathbb{R}}$ is a stochastic process over $\left(\Omega_{0}, \mathcal{F}_{0}\right)$ with state space $\mathbb{R}^{d}$.

For each $t \in \mathbb{R}$ define $\Theta_{t}: \Omega_{0} \rightarrow \Omega_{0}$ by the Formula

$$
\Theta_{t} u(s):=u(s+t)-u(t) ; \quad\left(u \in \Omega_{0}, s \in \mathbb{R}\right) .
$$

It can be easily verified that $\left(\Omega_{0}, \mathcal{F}_{0}, \Theta\right)$ is a dynamical system, called Wiener shift. Moreover, from (18) and (19), we obtain

$$
W_{s+t}(u)=W_{s}\left(\Theta_{t} u\right)+W_{t}(u) ; \quad\left(u \in \Omega_{0}, s, t \in \mathbb{R}\right)
$$

In other words $W$ is a $\Theta$-helix, called also Wiener helix.

By the well definitions, $W$ separates the points of $\Omega_{0}$ and generates $\mathcal{F}_{0}$. The following result is also a trivial consequence of Proposition 1.

Corollary 2 The Wiener shift $\Theta$ is the unique $D S$ for which $W$ is an helix.

\section{Remarks 4}

(1) Let $d=1$ and $\omega=I_{\mathbb{R}}$ the identity on $\mathbb{R}$. then for each $t \in \mathbb{R}, \Theta_{t} \omega=I_{\mathbb{R}}$ by the well definition of $\Theta$. Now, if there exists $h: \Omega_{0} \rightarrow \mathbb{R}$ measurable such that

$$
W_{t} \omega=h(\omega)-h\left(\Theta_{t} \omega\right) ; \quad(t \in \mathbb{R})
$$

then we must have $t=0$ by definition of $W_{t}$.

Hence, the Wiener helix can not be closed.

(2) From the definition of the Wiener helix $\left(W_{t}\right)$ (relation (18)), we have

$$
\frac{1}{s}\left(W_{t+s} \omega-W_{t} \omega\right)=\frac{1}{s}(\omega(t+s)-\omega(t))
$$

Hence, the Wiener helix can not be differentiable (It suffices to take any non-differentiable function $\left.\omega \in \Omega_{0}\right)$.

(3) In fact, it is known that $\left(W_{t}\right)$ is almost nowhere differentiable (cf. [2] for example).

\subsection{Coordinates-map}

Let $(\Omega, \mathcal{F})$ be a measurable space and let $H:=\left(H_{t}\right)_{t \in \mathbb{R}}$ be a stochastic process on $(\Omega, \mathcal{F})$ such that $H_{0}(\omega)=0$ for each $\omega \in \Omega$. The coordinates-map of $H$, is defined by

$$
\kappa(\omega):=\left(H_{t}(\omega)\right)_{t \in \mathbb{R}} ; \quad(\omega \in \Omega)
$$

Notice that $\kappa$ transforms $\Omega$ on $\Omega_{0}$. Moreover, by the well definitions of $\kappa$ and $W$, we have

$$
H_{t}(\omega)=W_{t}(\kappa(\omega)) ; \quad(t \in \mathbb{R}, \omega \in \Omega)
$$


Notice also that

\section{Lemma 2}

(1) $H$ is separating if and only if $\kappa$ is injective.

(2) If $H$ generates $\mathcal{F}$ then $\kappa$ is $\left(\mathcal{F}, \mathcal{F}_{0}\right)$-measurable.

Proof. The first assertion is straightforward.

Let $t_{1}, \ldots, t_{n} \in \mathbb{R}$ and $A_{1}, \ldots, A_{n} \in \mathcal{B}_{d}$. From (21) and (22), we have immediately

$$
\kappa^{-1}\left[W_{t_{1}} \in A_{1}, \ldots, W_{t_{1}} \in A_{1}\right]=\left[H_{t_{1}} \in A_{1}, \ldots, H_{t_{1}} \in A_{1}\right] .
$$

Hence $\kappa$ is $\left(\mathcal{F}, \mathcal{F}_{0}\right)$-measurable since $W$ generates $\mathcal{F}_{0}$ and $H$ generates $\mathcal{F}$.

Example 1 Let $\left(\Omega_{0}, \mathcal{F}_{0}, \Theta, W\right)$ be the Wiener helix and let $\Omega_{0}^{c}:=\left\{u \in \Omega_{0}: u\right.$ is continuous $\}$. Then $\Omega_{0}^{c}$ is $\Theta$-invariant and therefore, the restriction of $W$ on $\Omega_{0}^{c}$ is an helix (cf. Remark 1.3). It is called the continuous Wiener helix and is denoted by $\left(\Omega_{0}^{c}, \mathcal{F}_{0}^{c}, \Theta, W\right)$.

However, the coordinates-map, in this case, is exactly the canonical injection $i: \Omega_{0}^{c} \rightarrow \Omega_{0}$.

This example proves that, the coordinates-map may be not surjective.

Theorem 2 Let $(\Omega, \mathcal{F})$ be a measurable space and let $H$ be a stochastic process which separates the points of $\Omega$ and generates $\mathcal{F}$. Then $H$ is an helix if and only if $\kappa(\Omega)$ is $\Theta$-invariant.

Proof. Suppose first that $H$ is an helix which separates the points of $\Omega$ and generates $\mathcal{F}$. Let $\Phi$ be the unique DS on $(\Omega, \mathcal{F})$ (defined by Proposition 1 ) for which $H$ is an helix.

Let $\omega \in \Omega$ and $s, t \in \mathbb{R}$ then by (3), (21) and (22) we have

$$
\begin{aligned}
\kappa\left(\Phi_{t}(\omega)\right)(s) & =H_{s}\left(\Phi_{t}(\omega)\right) \\
& =H_{s+t}(\omega)-H_{t}(\omega) \\
& =W_{s+t}(\kappa(\omega))-W_{t}(\kappa(\omega)) \\
& =W_{s}\left(\Theta_{t}(\kappa(\omega))\right) \\
& =\Theta_{t}(\kappa(\omega))(s) .
\end{aligned}
$$

Hence $\kappa$ is an SD-morphism and therefore the restriction of $\Theta$ to $\kappa(\Omega)$ is a DS by Lemma 1 , since $\kappa$ is injective. En particular, $\kappa(\Omega)$ is $\Theta$-invariant. In fact the coordinates-map $\kappa$ is an SD-isomorphism between $\Omega$ and $\kappa(\Omega)$. Conversely, suppose that $\kappa(\Omega)$ is $\Theta$-invariant then, the restriction of $\Theta$ on $\kappa(\Omega)$ is a DS. On the other hand, since $H$ separates the points of $\Omega, \kappa$ must be injective and hence $\kappa: \Omega \rightarrow \kappa(\Omega)$ is one-to-one. We may also define

$$
\Phi_{t}(\omega):=\kappa^{-1}\left(\Theta_{t}(\kappa(\omega))\right) ; \quad(t \in \mathbb{R}, \omega \in \Omega)
$$

$\Phi_{t}: \Omega \rightarrow \Omega$ is well defined, since $\Theta(\kappa(\omega)) \in \kappa(\Omega)$ by the $\Theta$-invariance of $\kappa(\Omega)$. Notice that (23) is equivalent to

$$
\kappa\left(\Phi_{t}(\omega)\right)=\Theta_{t}(\kappa(\omega)) ; \quad(t \in \mathbb{R}, \omega \in \Omega) .
$$

Therefore by (22), (24) and (20), we have

$$
H_{s} \Phi_{t}=W_{s} \kappa \Phi_{t}=W_{s} \Theta_{t} \kappa=\left(W_{s+t}-W_{t}\right) \kappa=H_{s+t}-H_{t}
$$

for all $s, t \in \mathbb{R}$. This means that (3) is fulfilled. Since $H$ generates $\mathcal{F}$, we deduce as in the proof of Lemma 2 , that $\Phi_{t}$ is measurable and we conclude that $H$ is an helix. 
Corollary 3 Let $(\Omega, \mathcal{F}, \Phi, H)$ be an helix. If $H$ separates the points of $\Omega$ and generates $\mathcal{F}$, then $H$ is conjugate to the Winer helix $\left(\Omega_{0}, \mathcal{F}_{0}, \Theta, W\right)$.

\subsection{Linear transformations of helices}

Let $(\Omega, \mathcal{F}, \Phi)$ be a DS and let $H$ be a $\Phi$-helix on $\mathbb{R}^{d}$. If $f: \mathbb{R}^{d} \rightarrow \mathbb{R}^{n}$ is a linear then, $f \circ H$ is a $\Phi$-helix on $\mathbb{R}^{n}$.

In what follows, we deal with the converse problem: It turns out that if $H$ is "rich enough" and if $f \circ H$ is a $\Phi$-helix then $f$ must be linear.

Definition 3 Let $(\Omega, \mathcal{F}, \Phi)$ be a DS and let $H$ be a $\Phi$-helix on $\mathbb{R}^{d}$. $H$ is said to be connecting if for all $x, y \in \mathbb{R}^{d}$ there exists $\omega \in \Omega ; s, t \in \mathbb{R}$ such that $x=H_{s}(\omega)$ and $y=H_{t}(\omega)$.

\section{Examples 2}

(1) Suppose that $(\Omega, \mathcal{F})=(\mathbb{R}, \mathcal{B})$ and $\Phi_{t} \omega:=\omega+t$ for $\omega, t \in \mathbb{R}$. Then $(t, \omega) \mapsto t$ defines a $\Phi$-helix which is trivially connecting.

(2) Let $(\Omega, \mathcal{F}, \Phi)$ be a DS and let $h: \mathbb{R}^{d} \rightarrow \mathbb{R}^{d}$ be a bounded Borel function. Then $(t, \omega) \mapsto h(\omega)-h\left(\Phi_{t} \omega\right)$ defines a $\Phi$-helix which is not connecting. Indeed, if $M$ is such that $|f(x)| \leq M$ for each $x \in \mathbb{R}^{d}$, then there exists no $t \in \mathbb{R}$ and $\omega \in \Omega$ such that $z=H_{t}(\omega)$ if $|z| \geq 2 M+1$.

(3) Let $x, y \in \mathbb{R}^{d}$, then there exists $\omega: \mathbb{R} \rightarrow \mathbb{R}^{d}$ and $s, t \in \mathbb{R}$ such that $\omega(0)=0, \omega(s)=x$ and $\omega(t)=y$. Therefore the Wiener helix $\left(\Omega_{0}, \mathcal{F}_{0}, \Theta, W\right)$ is connecting.

Further, $\omega$ can be chosen to be continuous. Hence the continuous Wiener helix $\left(\Omega_{0}^{c}, \mathcal{F}_{0}^{c}, \Theta, W\right)$ is also connecting.

Theorem 3 Let $(\Omega, \mathcal{F}, \Phi)$ be a $D S, H$ a connecting $\Phi$-helix on $\mathbb{R}^{d}$ and let $f: \mathbb{R}^{d} \rightarrow \mathbb{R}^{n}$ be a Borel function. The following statements are equivalent

(1) $f \circ H$ is a $\Phi$-helix.

(2) $f$ is linear.

Proof: If $f$ is linear then obviously $f \circ H$ is a $\Phi$-helix whenever $H$ is a $\Phi$-helix.

Conversely, let $x, y \in \mathbb{R}^{d}$. Since $H$ is connecting, then there exists $\omega \in \Omega$ and $s, t \in \mathbb{R}$ such that

$$
H_{s}(\omega)=x ; \quad H_{t}(\omega)=y
$$

Applying (3) for the two $\Phi$-helices $H$ and $f(H)$, we obtain

$$
f\left(H_{s-t}\left(\Phi_{t} \omega\right)\right)=f\left(H_{s}(\omega)-H_{t}(\omega)\right)
$$

and

$$
f\left(H_{s-t}\left(\Phi_{t} \omega\right)\right)=f\left(H_{s}(\omega)\right)-f\left(H_{t}(\omega)\right)
$$

Combining (25), (26) and (27), we deduce that

$$
f(x-y)=f(x)-f(y) ; \quad\left(x, y \in \mathbb{R}^{d}\right) .
$$

In other words, $f$ is a Borel solution of the Cauchy equation $(28)$. We conclude by a classical result (cf. $[1,10]$ for example) that $f$ is linear.

Remark 5 If $H$ is not separating, it is clear how to construct a non linear function $f$ such that $f \circ H$ is an helix. Indeed, it suffices to start with a linear map $f$ (therefore $f \circ H$ will be an helix) and to change the value of $f$ on some points of $\mathbb{R}^{n}$, which are not reached by the map $H$. 


\subsection{Helices and cocycles}

Let $(\Omega, \mathcal{F}, \Phi)$ be a DS and let $(G, *)$ be a group with neutral element $e_{G}$.

An abstract $\Phi$-cocycle over the $\operatorname{DS} \Phi$, is a mapping $C: \mathbb{R} \times \Omega \times G \rightarrow G ;(t, \omega) \mapsto C(t, \omega)$ such that the family $C(t, \omega):=C(t, \omega,):. G \rightarrow G$, satisfies the cocycle equation, i.e.: For $s, t \in \mathbb{R}, \omega \in \Omega$

$$
C(0, \omega)=e_{G}, \quad C(s+t, \omega)=C\left(s, \theta_{t} \omega\right) * C(t, \omega)
$$

In particular,

(1) If $(G, *)=(] 0, \infty[, \times)$, then $C$ is called a multiplicative $\Phi$-cocycle. This equation is involved in many topics: Information without probability, abstract automat, lattice semigroups,... (cf. [6] and the related references).

(2) If $(G, *)=\left(\mathbb{R}^{d},+\right)$, then $C$ is called additive $\Phi$-cocycle or $\Phi$-helix on $\mathbb{R}^{d}$ (notice that (29) is equivalent to (3) in this case). The Wiener helix is considered as an important example of additive cocycle. It plays a fundamental role in the theory of stochastic integration and stochastic differential equations (cf. [2] and the related references).

(3) If $(G, *)=\left(G\left(\mathbb{R}^{d}\right), \circ\right)$ the group of self-mappings $\left\{f: \mathbb{R}^{d} \rightarrow \mathbb{R}^{d}\right\}$, then $C$ is said to be a cocycle on $\mathbb{R}^{d}$. In this case, $(\Phi, C)$ is the so called random dynamical system on $\mathbb{R}^{d}$. In particular, $C$ is a model of solutions of random iteration equations, random differential equations and stochastic differential equations (cf. [2] Part 1 and the related references).

Let $C$ be a cocycle on $\mathbb{R}^{d}$, over a DS $\Phi$. An associated random fix point is a random vector $Y: \Omega \rightarrow \mathbb{R}^{d}$ such that

$$
C(t, \omega) Y(\omega)=Y\left(\Phi_{t} \omega\right) ; \quad(t \in \mathbb{R}, \omega \in \Omega)
$$

(cf. [8] and the related references). Random fix points are exactly the stationary solutions of the cocycle equation on $\mathbb{R}^{d}$.

Let $(\Omega, \mathcal{F}, \Phi)$ be a DS, let $H$ be a $\Phi$-helix on $\mathbb{R}^{d}$ then

$$
\left.C(t, \omega) x:=H_{t}(\omega)\right)+x ; \quad\left(t \in \mathbb{R}, \omega \in \Omega, x \in \mathbb{R}^{d}\right)
$$

defines a $\Phi$-cocycle on $\mathbb{R}^{d}$. In this case the following fact can be easily verified: $C$ admits a random fix point if and only if $H$ is a closed $\Phi$-helix.

By Theorem 3 and Remark 1.1, we may replace $H$ in (31) by $f(a U+b V)$ where $a, b \in \mathbb{R}$ and $U, V$ are two $\Phi$-helices and $f$ is a linear mapping.

Some naturals questions arise:

(1) Are there other transformations of helices in order to obtain cocycles?

(2) Does there exist cocycles which are not generated by helices?

The following two remarks are classical in the framework of random dynamical systems (cf. [2]).

(1) In fact, the Wiener helix satisfies some additional properties (semi-martingale...) which allow to define the stochastic integral with respect to $W$, namely $\int_{0}^{t} f(\omega, s) d W_{s}(\omega)$ (cf. [9], Chap. III and IV). Therefore, the following stochastic differential equation may be also defined (cf. [9], Chap. V).

$$
d X_{t}=b\left(t, X_{t}\right) d t+\sigma\left(t, X_{t}\right) d W_{t} ; \quad X_{0}=x . \in \mathbb{R}^{d}
$$

Under some classical conditions on $b$ and $\sigma,(32)$ admits a unique solution denoted by $\varphi(t, \omega) x$. Moreover $(t, \omega, x) \rightarrow \varphi(t, \omega) x$ defines a cocycle on $\mathbb{R}^{d}$ over the Wiener shift $\Theta$ (cf. [2], Chap 2.3).

In particular such cocycles are also generated by helices. 
(2) Let $(\Omega, \mathcal{F}, \Phi)$ be a measurable dynamical system and let $f: \Omega \times \mathbb{R}^{d} \rightarrow \mathbb{R}^{d}$ be a measurable function. We may associate the random differential equation

$$
d X_{t}(\omega)=f\left(\Phi_{t} \omega, X_{t}\right) d t ; \quad X_{0}(\omega)=x \in \mathbb{R}^{d}
$$

Under some convenient conditions on $f,(33)$ admits a unique solution $\varphi(t, \omega) x$ which defines also a cocycle on $\mathbb{R}^{d}$ over the DS $\Phi$ (cf. [2], Chap 2.2).

However, such cocycles are not generated by helices.

Let $\Phi: \mathbb{R} \times \Omega \rightarrow \Omega$ and let $\varphi: \mathbb{R} \times \Omega \times \mathbb{R}^{d} \rightarrow \mathbb{R}^{d}$ be two maps. The associated skew product $\Lambda$ is defined by

$$
\begin{aligned}
& \Lambda: \quad \mathbb{R} \times \Omega \times \mathbb{R}^{d} \rightarrow \quad \Omega \times \mathbb{R}^{d} \\
& (t, \omega, x) \quad \rightarrow \quad\left(\theta_{t} \omega, \varphi(t, \omega) x\right) .
\end{aligned}
$$

By the well definitions, the following statements are equivalent:

(a) $\Phi$ is a DS on $\Omega$ and $\varphi$ is a cocycle over $\Phi$ on $\mathbb{R}^{d}$.

(b) $\Lambda$ is a DS on $\Omega \times \mathbb{R}^{d}$.

A $\Lambda$-helix is also a stochastic process $K=\left(K_{t}\right)_{t \in \mathbb{R}}$ such that $K_{t}: \Omega \times \mathbb{R}^{d} \rightarrow \mathbb{R}^{d}, K_{0}(\omega, x)=0$ and

$$
K_{s+t}(\omega, x)=K_{s}\left(\Phi_{t} \omega, \varphi(\omega, t) x\right)+K_{t}(\omega, x) .
$$

For example $K_{t}(\omega, x):=\varphi(t, \omega) x-x$ is a $\Lambda$-helix.

We conclude this paper by introducing a new notion.

Let $(\Omega, \mathcal{F}, \Phi)$ be a measurable dynamical system. An associated bi-helix is a pair $(H, K)$ where

(1) $H: \mathbb{R} \times \Omega \rightarrow \mathbb{R}^{d},(t, \omega) \rightarrow H_{t}(\omega)$ is an helix over $\Phi$,

(2) $K: \mathbb{R} \times \Omega \times \mathbb{R}^{d} \rightarrow \mathbb{R}^{d},(t, \omega, x) \mapsto K_{t}(\omega, x)$ is an helix over the DS $\Lambda$ defined by $\Lambda_{t}(\omega, x):=\left(\Phi_{t} \omega, H_{t}(\omega)+\right.$ $x)$.

Hence by (3) and (34), $(H, K)$ satisfies the coupling system

$$
\begin{cases}H_{0}(\omega)=0, & H_{s+t}(\omega)=H_{s}\left(\Phi_{t} \omega\right)+H_{t}(\omega) \\ K_{0}(\omega, x)=0, & K_{s+t}(\omega, x)=K_{s}\left(\Phi_{t} \omega, H_{t}(\omega)+x\right)+K_{t}(\omega, x) .\end{cases}
$$

Although (35) is defined in a theoretical way, it seems that a new light can be shed on this system.

Remark 6 Notice that cocycle equations have been studied during the last years in different situations of rings of power series, in a series of papers by H. Fripertinger and L. Reich, with a somewhat different terminology. In their proofs, also, the helix plays a role (cf. [3] and the related references).

\section{REFERENCES}

[1] Aczel J.: Lectures on functional equations and their applications, Academic Press Inc., New York, (1966).

[2] Arnold L.: Random Dynamical Systems, Springer-Verlag, Berlin (1998).

[3] Fripertinger H. \& Reich L.: On The formal first cocycle equation for iteration groups of type II, To appear in this volume.

[4] Hajek, O.: Dynamical Systems in the Plane, Academic Press, London, New York (1968).

[5] Hmissi F. \& Hmissi, M.: On Dynamical Systems generated by Homogeneous Processes, Grazer Math. Berichte 350, 144-155 (2006).

[6] Hmissi, M.: Sur les solutions globales de l'équation des cocycles, Aequ. Math. 45, 195-206 (1993).

[7] Kolmogorov, A.N.: The Wiener helix and other interesting curves in Hilbert space, Dokl. Acad. Nauk. 26,115118 (in Russian) (1940).

[8] Schmalfuss B.: A Random Fix Point Theorem Based on Liapunov Exponents, Random and Computational Dynamics 4(4), 257-268 (1996). 
[9] Oksendal B.: Stochastic Differential Equations, Universitext, Springer-Verlag, Sixth edition (2006).

[10] Wilansky, A: Additive functions, Lectures on calculus, ed. by K. May, Holden-Day, San Francisco, 97-124 (1967). 